\title{
Hepatic histopathological changes and dysfunction in primates following exposure to organic arsenic diphenylarsinic acid
}

\author{
Tomoyuki Masuda ${ }^{1,2, *}$, Kazuhiro Ishii1,*, Yukio Morishita ${ }^{3}$, Nobuaki Iwasaki ${ }^{4}$, \\ Yasuyuki Shibata ${ }^{5}$ and Akira Tamaoka ${ }^{1}$
}

${ }^{1}$ Department of Neurology, Faculty of Medicine, University of Tsukuba, 1-1-1 Tennodai, Tsukuba, Ibaraki 305-8575, Japan ${ }^{2}$ Department of Neurobiology, Faculty of Medicine, University of Tsukuba, 1-1-1 Tennodai, Tsukuba, Ibaraki 305-8577, Ibaraki, Japan

${ }^{3}$ Department of Diagnostic Pathology, Tokyo Medical University Ibaraki Medical Center, 3-20-1 Chuo, Ami, Ibaraki 300-0395, Japan

${ }^{4}$ Department of Pediatrics, Ibaraki Prefectural University of Health Sciences, 4669-2 Ami, Ami, Ibaraki 300-0331, Japan ${ }^{5}$ Center for Environmental Measurement and Analysis, National Institute for Environmental Studies, 16-2 Onogawa, Tsukuba, Ibaraki 305-8506, Japan

(Received December 26, 2017; Accepted February 14, 2018)

\begin{abstract}
Organic arsenic diphenylarsinic acid (DPAA[V]) accumulates at high concentrations in the liver of primates after its subchronic administration. However, no studies on the hepatic effects of organic arsenic compounds, including DPAA(V), on primates have been reported to date. To clarify the toxicokinetics of DPAA $(\mathrm{V})$ in the liver of primates, hepatic tissue specimens were collected from cynomolgus monkeys $(\mathrm{n}=32)$ at $5,29,170$, and 339 days after repeated administration of DPAA(V) for 28 days. Four histopathological changes in the specimens were observed and pathologically evaluated. Atypical ductular proliferation was found in the DPAA(V)-exposed liver throughout the period. Inflammatory cell infiltration in Glisson's capsules and lipid droplets were seen at earlier periods after administration. Conversely, inflammatory cell infiltration in liver lobules was seen later after administration. In this experiment, we did not confirm the hepatic dysfunction of DPAA(V)-exposed monkeys by blood chemistry tests. To compensate for this, we further investigated the blood from a patient who exhibited several neurological symptoms after DPAA(V) exposure. Her blood chemistry test values for aspartate transaminase, alanine transaminase, and lactate dehydrogenase were elevated, suggesting that her liver may have been damaged by DPAA(V) exposure. Together, these findings suggest that the accumulation of DPAA(V) may induce differential histopathological changes in primate hepatocytes, resulting in decreased liver function. This is the first report to investigate the liver of primates pathologically after exposure to organic arsenic DPAA(V). Our findings will help expand our knowledge regarding the effect of $\operatorname{DPAA}(\mathrm{V})$ on the liver of primates.
\end{abstract}

Key words: Macaca fascicularis, Primate, Phenyl arsenic compound, Long-term exposure, Hepatic pathology, Hepatic damage

\section{INTRODUCTION}

Organic arsenic tends to be less toxic than inorganic arsenic (Shi et al., 2004; Valko et al., 2005). Among organic arsenic compounds, monomethylarsonic acid and dimethylarsinic acid are known to be metabolites of inorganic arsenicals. The metabolism and genotoxicity of these two organic arsenic compounds have been well studied since the early 1980s (Cohen et al., 2006). However, organic arsenic diphenylarsinic acid (DPAA[V]) is not a natural product. Our knowledge of the toxicokinetics of DPAA(V) was therefore quite limited until 2003.

In the 2003 poisoning incident involving DPAA(V) in Kamisu, Ibaraki Prefecture, Japan, the residents suffered

Correspondence: Tomoyuki Masuda (E-mail: tmasu@md.tsukuba.ac.jp)

*These authors equally contributed to this work. 
from unexplained symptoms restricted to the central nervous system (CNS) (Ishii et al., 2004). DPAA(V) in the drinking-water wells was identified as the cause of these symptoms (Ishii et al., 2004). After this incident, both human and rodent studies were conducted to reveal the effects of DPAA(V) on the CNS. Analyses of the cerebral blood flow by single-photon emission computed tomography in DPAA(V)-exposed residents revealed a decrease in the blood flow in the occipital lobe and cerebellar vermis (Ishii and Tamaoka, 2015). Rodent studies suggest that the accumulation of DPAA(V) in the CNS influences both behavior and learning ability (Negishi et al., 2013; Ozone et al., 2010; Umezu et al., 2012).

We previously showed that DPAA(V) accumulates at high concentrations not only in the CNS but also in the liver after its oral administration to monkeys (Masuda et al., 2017). In general, arsenic compounds are known to be toxic to hepatocytes (Rehman and Naranmandura, 2012; Xie et al., 2004). The hepatic effects of organic arsenic compounds on rodents have already been studied (Ahmad et al., 1999; Siewicki, 1981; Yamaguchi et al., 2017). However, the influence of organic arsenic compounds on the liver of primates is largely unknown (Jomova et al., 2011). There are apparent differences between rodents and primates in the structure and metabolism of the liver. First, from a microscopic point of view, there is a distinct structure of liver lobules in humans and non-human primates, whereas the liver lobules in rodents are not clearly separated because there are less interlobular connective tissues (Glisson's capsules) in rodents than in primates (MIT OpenCourseWare, 2005). At the level of gross anatomy, the human liver is divided into eight lobes, whereas the rat liver is composed of only four lobes (Vdoviaková et al., 2016). Furthermore, the rat liver does not have a gallbladder. Therefore, rat bile is directly released into the bile duct from the liver (Vdoviaková et al., 2016). From a metabolic point of view, a comparative study on the liver transcriptome further shows that there is a functional variance of genes expressed in the liver between rats and humans, which reflects the metabolic difference between them (Yu et al., 2010). Thus, the exact hepatic effect of organic arsenic compounds on primates cannot be determined by studying the effects on rodents.

In this study, we reported for the first time the influence of organic arsenic DPAA(V) on the liver of primates. We examined the effect of DPAA(V) on the liver of primates by preparing liver specimens of 32 cynomolgus monkeys following DPAA(V) administration. During 5-339 days after the DPAA(V) administration, we observed differential histopathological changes in their hepatocytes. In addition, we found an eight-year-old girl who had elevated concentrations of liver enzymes and neurological symptoms in the human exposure cohort study in Kamisu, suggesting that the accumulation of DPAA(V) may induce histopathological changes and dysfunction in primate hepatocytes.

\section{MATERIALS AND METHODS}

\section{Arsenic}

DPAA(V) (>99.0\%) was purchased from Tri Chemical Laboratories (Yamanashi, Japan) and stored at $4^{\circ} \mathrm{C}$ in the dark.

\section{Experimental animals, housing, and husbandry}

Thirty-two purpose-bred cynomolgus monkeys (Macaca fascicularis) of Chinese origin were obtained from Hamri Co., Ltd. (Ibaraki, Japan). At the start of the study, they were 36-48 months old with a mean body weight of $3.15 \mathrm{~kg}$. During the study period, each monkey was housed in an individual stainless steel cage $(680 \times 658 \times 770 \mathrm{~mm})$, at $24.3-23.9^{\circ} \mathrm{C}$ with a relative humidity of $45.4 \%-82.2 \%( \pm 40 \%)$ and a $12-\mathrm{hr}$ light $/ 12-\mathrm{hr}$ dark cycle. The animals received CLEA Old World Monkey Diet (CMK-2; CLEA Japan, Inc., Tokyo, Japan) twice a day. All of them had free access to tap water. The food intake by the monkeys was checked daily throughout the study. The body weights and clinical observations were recorded prior to the administration of DPAA(V).

\section{Experimental design and collection of hepatic tissues from the monkeys}

DPAA(V) was dissolved in distilled water at a concentration of $10 \mathrm{mg} / \mathrm{mL}$, and the $\mathrm{pH}$ was adjusted to 7.0 with $1 \mathrm{~N} \mathrm{NaOH}$. Each monkey in the experimental group $(\mathrm{n}=28)$ was orally administered DPAA(V) via a nasogastric tube at a dose of $1.0 \mathrm{mg} / \mathrm{kg} /$ day (Masuda et al., 2017). Instead of DPAA(V), distilled water was administered to the control group $(n=4)$. After the repeated administration of DPAA(V) for 28 days, the monkeys in the experimental group were divided into 4 subgroups: a 5-day subgroup $(n=6), 29$-day subgroup $(n=6)$, 170-day subgroup $(\mathrm{n}=8)$, and a 339-day subgroup $(\mathrm{n}=8)$. The monkeys in these subgroups were euthanized at 5, 29, 170, and 339 days after the last administration (Fig. 1). After euthanasia, the hepatic tissue specimens were excised and fixed in a fixative containing $4 \%$ paraformaldehyde, processed by standard procedures, embedded in paraffin, sectioned, and stained with hematoxylin and eosin for light microscopy examination. DPAA(V) administration to monkeys and tissue sampling were performed at LSI Medience Corporation (Tokyo, Japan) in accordance with 
Hepatic dysfunction in primates after DPAA exposure

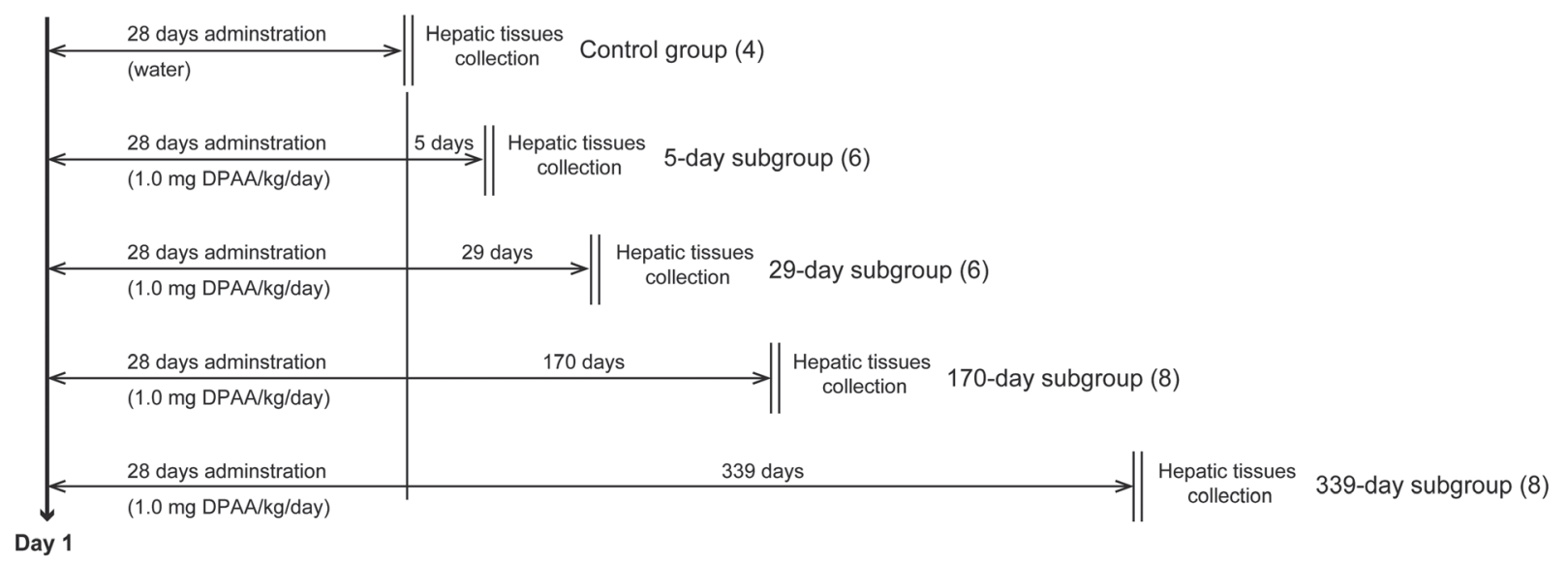

Fig. 1. Experimental design of the study using cynomolgus monkeys. Hepatic tissue specimens were collected from the control group and experimental subgroups at 5, 29, 170, and 339 days after the last administration of DPAA(V). The number in brackets indicates the number of monkeys examined.

the requirements of the Animals (Scientific Procedures) Act 1986. All experiments were conducted in compliance with guidelines within the "Guide for the Care and Use of Laboratory Animals (8th ed., National Research Council of National Academies)".

\section{Histological staging and criteria for scoring of hepatic tissues in monkeys}

A histological staging system to clarify the histopathological changes in the liver induced by arsenic compounds has not been proposed yet. The histopathological changes in the hepatic tissue specimens following DPAA(V) exposure showed histopathology that approximated that of human primary biliary cholangitis (PBC). Recently, new histopathological staging systems for $\mathrm{PBC}$ have been proposed, in which PBC is histologically classified into four stages (Hiramatsu et al., 2006). Using these systems as a reference, we proposed a new histopathological staging system to evaluate the effect of arsenic on the liver of primates. In this staging system, the following four histopathological changes were pathologically evaluated: atypical ductular proliferation, inflammatory cell infiltration in Glisson's capsules, inflammatory cell infiltration in liver lobules, and lipid droplets (LDs) in hepatocytes. These changes were given a staging score of 3, 2, 1 , or 0 in accordance with the criteria shown in Table 1. As for atypical ductular proliferation, a score of 0 means no atypical ductular proliferation. A score of 1 means 1 atypical ductular proliferation per section. Scores of 2 and 3 are characterized by 2-3 and over 4 atypical ductular proliferations per section, respectively. As for inflammatory cell infiltration in Glisson's capsules, a score of 0 indicates no inflammatory cell infiltration in them. Scores of 1,2 and 3 indicate the presence of inflammatory cell infiltration in less than $1 / 3,1 / 3-2 / 3$ and more than $2 / 3$ of the Glisson's capsules, respectively. As for inflammatory cell infiltration in liver lobules, a score of 0 indicates no inflammatory cell infiltration in liver lobules. Scores of 1, 2 and 3 refer to obvious inflammatory cell infiltration in less than $1 / 3,1 / 3-2 / 3$ and more than $2 / 3$ of liver lobules, respectively. As for LDs in hepatocytes, a score of 0 indicates no LDs in periportal hepatocytes. Scores of 1, 2 and 3 mean that 1-4, 5-19 and over 20 LDs were seen per periportal area in hepatocytes, respectively. All pathological observations were performed in a blind fashion by three researchers.

\section{Statistical analyses}

The Kruskal-Wallis test (nonparametric statistics) was used to analyze the heteroscedastic data. The Tukey-Kramer test (parametric statistics) was used for homoscedastic data. Statistical analyses were performed by using the JMP software program (version 5.12-J; SAS Institute Inc., Cary, NC, USA). $P$ values $<0.05$ were considered statistically significant.

\section{A health examination of and liver function test in a human}

A patient (an eight-year-old girl) agreed to undergo physical, dermatological, and neurological examinations. Her peripheral blood was collected, and the concentrations of aspartate transaminase (AST), alanine transaminase (ALT), and lactate dehydrogenase (LDH) were measured in the University of Tsukuba Hospital, Japan. 
T. Masuda et al.

Table 1. Evaluation of 4 histological changes and criteria for their scoring following DPAA exposure.

\begin{tabular}{lcccc}
\hline Histological changes & \multicolumn{4}{c}{ Score } \\
\cline { 2 - 5 } Atypical ductular proliferation & 0 & 1 & 2 & 3 \\
Inflammatory cell infiltration in Glisson's & 0 per section & 1 per section & $2-3$ per section & $>4$ per section \\
capsules & Absent & $<1 / 3$ Glisson's & $1 / 3-2 / 3$ Glisson's & $>2 / 3$ Glisson's \\
Inflammatory cell infiltration in liver lobules & Absent & $<1 / 3$ liver & $1 / 3-2 / 3$ liver & $>2 / 3$ liver \\
& capsules & lobules & lobules & lobules \\
Lipid droplets (LDs) in hepatocytes & periportal area & periportal area & periportal area & periportal area \\
\hline
\end{tabular}

The study was approved by the Ethics Committee of the University of Tsukuba. Informed consent was obtained from the patient in accordance with the rules of this committee.

\section{RESULTS}

\section{Histopathological changes in the hepatic tissues of monkeys after DPAA(V) exposure}

The maximum exposed dose of the subjects in the Kamisu cohort who developed CNS symptoms was estimated to be $0.93 \mathrm{mg} / \mathrm{kg} /$ day (Ishii et al., 2014). The monkeys in our study were therefore administered $1.0 \mathrm{mg} / \mathrm{kg} /$ day of DPAA(V) orally according to the schedule shown in Fig. 1 (Masuda et al., 2017). After euthanasia, the specimens of the liver in each experimental subgroup were prepared, and four histopathological changes were evaluated in accordance with the criteria (Fig. 2, Table 1). An increased rate of atypical ductular proliferation was observed from 5 to 339 days after the last administration (27/28 cases). A high rate of inflammatory cell infiltration in Glisson's capsules was also seen from 5 to 339 days (25/28 cases). However, inflammatory cell infiltration in liver lobules was scarcely detected at 5 days after the last administration ( $1 / 6$ cases), whereas a high rate of the infiltration was observed at 170 days $(7 / 8$ cases). Inflammatory infiltrates decreased by 339 days after the last administration (5/8 cases). LDs in hepatocytes were remarkably increased in number at 5 days $(5 / 6$ cases) and gradually decreased thereafter at 29-339 days (10/22 cases). No hepatocellular necrosis was seen in any time period. These findings suggest that subchronic exposure to DPAA(V) may have induced transient changes in the hepatic tissues of monkeys for a long duration after exposure.

\section{Semi-quantitative evaluation of histopathological changes in the hepatic tissues of monkeys after DPAA(V) exposure}

The four histopathological changes are represented as bar graphs in Fig. 3. The average scores for atypical ductular proliferation in all of the experimental subgroups were significantly higher than those in the control group (Fig. 3A, $p<0.01$, Tukey-Kramer test for parametric statistics). The average score for inflammatory cell (lymphocytes and eosinophils) infiltration in Glisson's capsules in the 5-day subgroup was significantly higher than that in the control group (Fig. 3B, $p=0.0147$, Tukey-Kramer test for parametric statistics). The average score for inflammatory cell infiltration in liver lobules in the 170-day subgroup was significantly higher than that in the control group (Fig. 3C, $p=0.0118$, KruskalWallis test for nonparametric statistics). The average score for LDs in hepatocytes in the 5-day subgroup was significantly higher than that in the control group (Fig. 3D, $p=0.0316$, unpaired Tukey-Kramer test for parametric statistics). Taken together, these results further indicate that subchronic exposure to DPAA(V) differentially led to obvious hepatocellular damages in primates.

\section{Human case}

An eight-year-old girl was admitted to our hospital a month after she had stopped drinking well water contaminated with DPAA(V). In the neurological examination, she exhibited several neurological symptoms, including mild dysmetria of both upper extremities, postural tremor, horizontal gaze nystagmus, and tandem gait difficulty. Her blood chemistry test values were within normal ranges except for the biochemical parameters of the liver function. Specifically, the test values were negative for anemia and showed a normal blood glucose level and no inflammation. However, the values of AST (normal value 8-40 U/L), ALT (normal value 4-40 U/L), and LDH (normal value 124-232 U/L) were elevated (AST, $61 \mathrm{U} / \mathrm{L}$; ALT, $50 \mathrm{U} / \mathrm{L}$; and LDH, $283 \mathrm{U} / \mathrm{L}$ ). These results suggest 

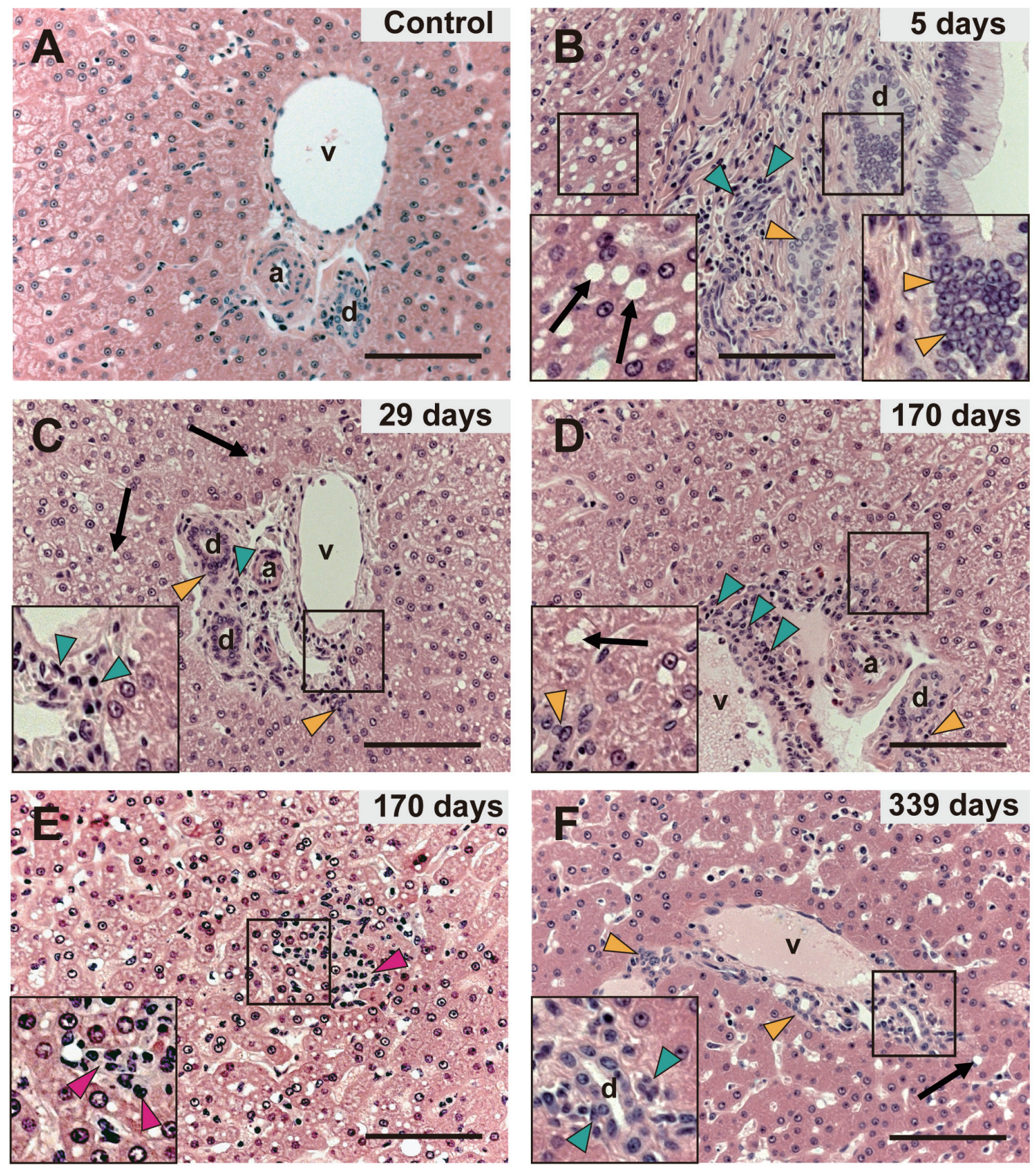

Fig. 2. Histopathological changes in the hepatic tissue specimens of cynomolgus monkeys induced by DPAA(V) administration. (A) This panel shows a hematoxylin and eosin-stained section of the control hepatic tissues. (B-F) These panels show sections from the experimental subgroups obtained at 5 days (B: Scores for atypical ductular proliferation, infiltration in Glisson's capsules and LDs were 2, 2 and 3, respectively), at 29 days (C: 2, 2 and 2, respectively), at 170 days (D, E: Scores for atypical ductular proliferation and LDs were 2 and 2, respectively [D] and the score for infiltration in liver lobules was 2 [E]), and at 339 days (F: Scores for atypical ductular proliferation and infiltration in Glisson's capsules were 2 and 2, respectively) after the last administration. The insets show higher-magnification views of the boxed areas. The orange arrowheads indicate atypical ductular proliferation; the green arrowheads, inflammatory cell (lymphocytes and eosinophils) infiltration in Glisson's capsules; the magenta arrowheads, inflammatory cell (lymphocytes and eosinophils) infiltration in liver lobules; and the arrows indicate LDs. a, interlobular artery; d, interlobular bile duct; v, portal vein. Scale bars, $100 \mu \mathrm{m}$. 
A Atypical ductular proliferation

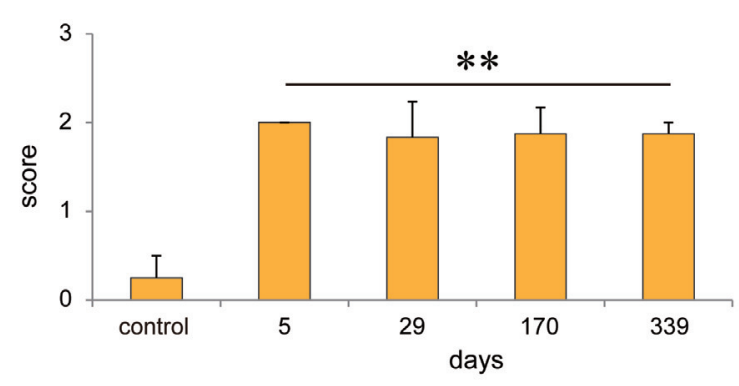

\section{Inflammatory cell infiltration in liver lobules}

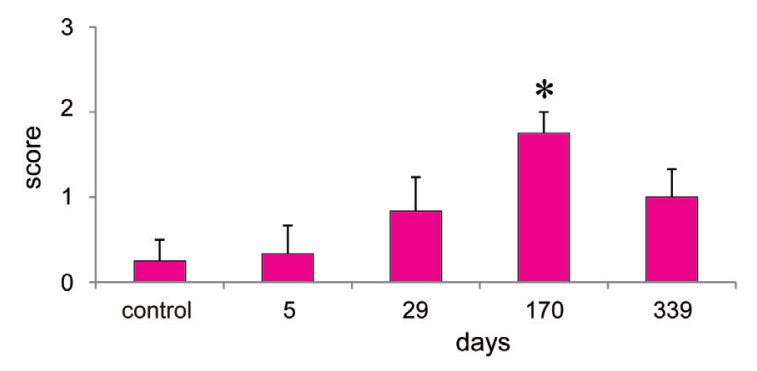

B Inflammatory cell infiltration in Glisson's capsules

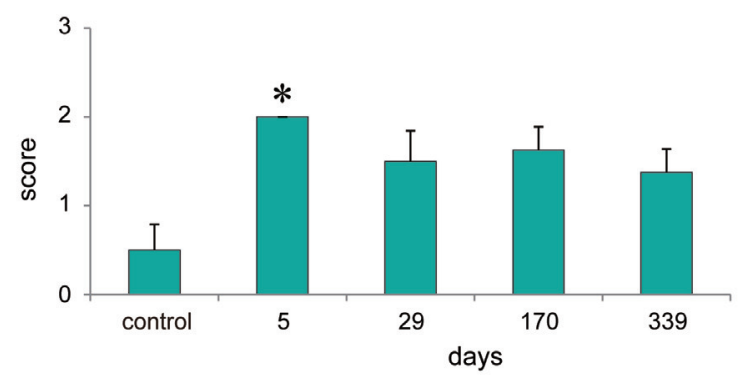

D Lipid droplets (LDs) in hepatocytes

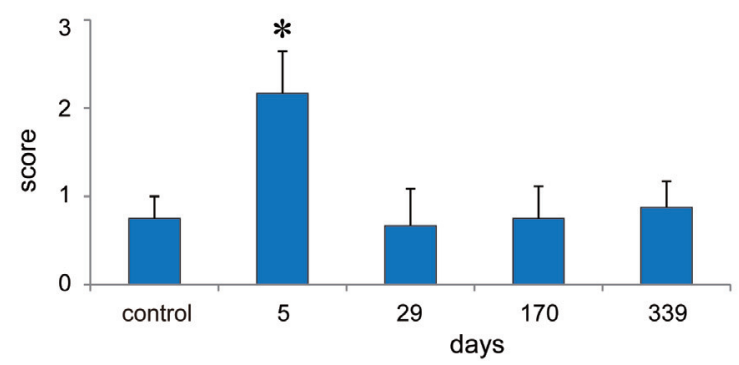

Fig. 3. Scoring of histopathological observations in the hepatic tissues of cynomolgus monkeys after the oral administration of DPAA(V). (A) The average scores for atypical ductular proliferation in all of the experimental subgroups were significantly higher than that in the control group. (B) The average score for inflammatory cell infiltration in Glisson's capsules in the 5-day subgroup was significantly higher than that in the control group. (C) The average score for inflammatory cell infiltration in liver lobules in the 170-day subgroup was significantly higher than that in the control group. (D) The average score for LDs in hepatocytes in the 5-day subgroup was significantly higher than that in the control group. The bars represent the mean + S.E.M. $* * p<0.01,{ }^{*} p<0.05$.

that her hepatocytes might have been damaged by the exposure to DPAA(V).

\section{DISCUSSION}

In this study, we found several differential histopathological changes in the hepatic tissues of monkeys at an exposure level comparable to that in the human exposure cohort. Furthermore, our examination in the human cohort showed that the single patient displayed hepatic dysfunction after the exposure to DPAA $(\mathrm{V})$.

We previously found that the average concentration of DPAA $(V)$ in the liver of the 5-day subgroup was significantly higher than that in the other tissues (555 ng As/g) (Masuda et al., 2017). However, the DPAA(V) accumulation decreased rapidly in the 29-day subgroup, and very little DPAA(V) was detected in the 170- and 339-day subgroups. Therefore, the histopathological changes observed in this study were likely induced by the increased accumulation of DPAA $(\mathrm{V})$ during the initial period. Because the blood chemistry test for the patient was performed at 1 month after the last exposure, her exposure level was deemed to have been equivalent to that of the 29-day subgroup of monkeys. Because inflammatory cell infiltration is known to be related to elevated levels of AST, ALT and LDH (Theise, 2014), the patient's hepatic tissues would be expected to exhibit inflammatory cell infiltration in Glisson's capsules to the same degree that observed in monkey hepatocytes of the 29-day subgroup. The limitation of this study is the lack of the blood samples from DPAA(V)-exposed monkeys. If we obtained the blood data, our speculation would be further strengthened.

Glutathione (GSH) is known to be abundant in the liver (Kaplowitz, 1981). GSH is conjugated to DPAA(V) by the enzyme called glutathione S-transferase (GST). As a result, the glutathione adduct of DPAA (V) [DPA-GS], 
Hepatic dysfunction in primates after DPAA exposure

which is known to be highly toxic in vitro, is synthesized in the liver (Ochi et al., 2006). DPAA(V) is thought to act on liver macrophages and to cause hepatitis by inducing the release of inflammatory cytokines by macrophages. However, it is unclear which of DPAA(V) and DPA-GS may actually act on liver macrophages to cause inflammation in vivo. In addition, we cannot determine whether the activity of the primate liver GST to conjugate to DPAA(V) may be higher than that of the rat GST or not, because the hepatic GST activity varies markedly depending on its substrate. When the substrate is dichloromethane or 1,2-dichloro-4-nitrobenzene, hepatic GST activity in rodents is very high compared with that in primates. However, in the case of 1-chloro-2, 4-dinitrobenzene, the result is opposite (Igarashi and Satoh, 1989; Thier et al., 1998). In a previous study using DPAA(V)-exposed rodents, no abnormalities other than intrahepatic bile duct hyperplasia in the hepatic tissues after DPAA(V) exposure were detected (Yamaguchi et al., 2017). We also observed no pathological changes in the liver of rats administered $1.2 \mathrm{mg} / \mathrm{kg}$ DPAA(V) (unpublished observation). Thus, we will advocate two hypotheses. The first hypothesis is based on the assumption that the DPAA(V)-conjugating activity of the primate GST may be higher than that of rodents and that DPA-GS may exert higher inflammatory cytokine-inducing activity than DPAA(V) in vivo. Under this assumption, we propose that the primate liver may produce more DPA-GS compared with the rodent one, which may result in hepatitis in primates. The second hypothesis is based on the assumption that the DPAA(V)conjugating activity of the primate GST may be lower than that of rodents and that DPAA(V) may exert higher inflammatory cytokine-inducing activity than DPA-GS in vivo. Under this assumption, we suggest that DPAA(V) may be maintained at a high level in the primate liver, which may result in hepatitis in primates. The progress of the future research may reveal which of these hypotheses is the correct one.

Inflammatory cytokines released by macrophages are known to induce not only acute inflammatory liver injury but also the development of hepatic steatosis (accumulation of LDs; Baeck et al., 2012). In other words, hepatitis (inflammatory cell infiltration) and LD accumulation are common phenomena that progress at the same time as was seen in the 29-day subgroup. However, the pathogenesis of hepatitis is multifactorial and is not necessarily accompanied by an increase in the number of LDs. In the absence of Galectin-3 known as a lectin family molecule, hepatitis declines, whereas hepatic fat deposition proceeds (Jeftic et al., 2015). This finding shows the example that both phenomena do not proceed at the same time.
Even in this study, hepatic fat deposition and inflammatory cell infiltration in the liver lobules did not occur at the same time. We speculate that this inflammation might have appeared later (170 days after the last administration) for some unknown reason.

In this study, the overproduction of LDs was observed in the specimens of the 5-day subgroup. Recent studies have revealed that LDs not only store lipids but have multiple functions in vivo. For example, Singh et al. (2009) clarified that LDs are decomposed by autophagy upon hunger and become a source of nutritional supplementation. Also, it has been found by another group that the formation of LDs enhances the formation of additional LDs through a positive feedback loop (Takahashi et al., 2013). As described above, the increase of LD number in the liver observed in this study was supposedly caused by inflammatory cytokines released by macrophages. However, we cannot exclude the possibility that LDs might play an active role against arsenic exposure.

In conclusion, we found transient histopathological changes in the hepatocytes of monkeys following DPAA(V) administration. These differential changes are likely correlated with the liver dysfunction in humans. Our findings may be useful for developing a future treatment strategy for patients who have been exposed to $\operatorname{DPAA}(\mathrm{V})$.

\section{ACKNOWLEDGMENTS}

This work was supported by JSPS KAKENHI Grant Nos. JP20390039, JP16590811 and JP15K08148 from the program Grants-in-Aid for Scientific Research of the MEXT, Japan, and by a Grant-in-Aid for research on Study (Toxic study group) of the Health Effects of DPAA supported by the Ministry of the Environment.

Conflict of interest---- The authors declare that there is no conflict of interest.

\section{REFERENCES}

Ahmad, S., Anderson, W.L. and Kitchin, K.T. (1999): Dimethylarsinic acid effects on DNA damage and oxidative stress related biochemical parameters in B6C3F1 mice. Cancer Lett., 139, 129-135.

Baeck, C., Wehr, A., Karlmark, K.R., Heymann, F., Vucur, M., Gassler, N., Huss, S., Klussmann, S., Eulberg, D., Luedde, T., Trautwein, C. and Tacke, F. (2012): Pharmacological inhibition of the chemokine CCL2 (MCP-1) diminishes liver macrophage infiltration and steatohepatitis in chronic hepatic injury. Gut, 61, 416-426.

Cohen, S.M., Arnold, L.L., Eldan, M., Lewis, A.S. and Beck, B.D. (2006): Methylated arsenicals: the implications of metabolism 
T. Masuda et al.

and carcinogenicity studies in rodents to human risk assessment. Crit. Rev. Toxicol., 36, 99-133.

Hiramatsu, K., Aoyama, H., Zen, Y., Aishima, S., Kitagawa, S. and Nakanuma, Y. (2006): Proposal of a new staging and grading system of the liver for primary biliary cirrhosis. Histopathology, 49, 466-478

Igarashi, T. and Satoh, T. (1989): Sex and species differences in glutathione S-transferase activities. Drug Metabol. Drug Interact., 7, 191-212.

Ishii, K., Tamaoka, A., Otsuka, F., Iwasaki, N., Shin, K., Matsui, A., Endo, G., Kumagai, Y., Ishii, T., Shoji, S., Ogata, T., Ishizaki, M., Doi, M. and Shimojo, N. (2004): Diphenylarsinic acid poisoning from chemical weapons in Kamisu, Japan. Ann. Neurol., 56, 741-745.

Ishii, K., Itoh, Y., Iwasaki, N., Shibata, Y. and Tamaoka, A. (2014): Detection of diphenylarsinic acid and its derivatives in human serum and cerebrospinal fluid. Clin. Chim. Acta, 431, 227-231.

Ishii, K. and Tamaoka, A. (2015): Ten-years records of organic arsenic (diphenylarsinic acid) poisoning: epidemiology, clinical feature, metabolism, and toxicity. Brain Nerve, 67, 5-18.

Jeftic, I., Jovicic, N., Pantic, J., Arsenijevic, N., Lukic, M.L. and Pejnovic, N. (2015): Galectin-3 ablation enhances liver steatosis, but attenuates inflammation and IL-33-Dependent fibrosis in obesogenic mouse Model of nonalcoholic steatohepatitis. Mol. Med., 21, 453-465.

Jomova, K., Jenisova, Z., Feszterova, M., Baros, S., Liska, J., Hudecova, D., Rhodes, C.J. and Valko, M. (2011): Arsenic: toxicity, oxidative stress and human disease. J. Appl. Toxicol., 31, 95-107.

Kaplowitz, N. (1981): The importance and regulation of hepatic glutathione. Yale J. Biol. Med., 54, 497-502.

Masuda, T., Ishii, K., Seto, Y., Hosoya, T., Tanaka, R., Nakayama, T., Iwasaki, N., Shibata, Y. and Tamaoka, A. (2017): Long-term accumulation of diphenylarsinic acid in the central nervous system of cynomolgus monkeys. Arch. Toxicol., 91, 2799-2812.

MIT OpenCourseWare. (2005): Liver anatomy and histology. https://ocw.mit.edu/courses/biological-engineering/20-450-molecular-and-cellular-pathophysiology-be-450-spring-2005/lecture-notes/ Accessed 28 Jan 2018.

Negishi, T., Matsunaga, Y., Kobayashi, Y., Hirano, S. and Tashiro, T. (2013): Developmental subchronic exposure to diphenylarsinic acid induced increased exploratory behavior, impaired learning behavior, and decreased cerebellar glutathione concentration in rats. Toxicol. Sci., 136, 478-486.

Ochi, T., Kinoshita, K., Suzuki, T., Miyazaki, K., Noguchi, A. and Kaise, T. (2006): The role of glutathione on the cytotoxic effects and cellular uptake of diphenylarsinic acid, a degradation product of chemical warfare agents. Arch. Toxicol., 80, 486-491.

Ozone, K., Ueno, S., Ishizaki, M. and Hayashi, O. (2010): Toxicity and oxidative stress induced by organic arsenical diphenylarsinic acid and inorganic arsenicals and their effects on spatial learning ability in mice. J. Health Sci., 56, 517-526.

Rehman, K. and Naranmandura, H. (2012): Arsenic metabolism and thioarsenicals. Metallomics, 4, 881-892.

Shi, H., Shi, X. and Liu, K.J. (2004): Oxidative mechanism of arsenic toxicity and carcinogenesis. Mol. Cell. Biochem., 255, 67-78.

Siewicki, T.C. (1981): Tissue retention of arsenic in rats fed witch founder or cacodylic acid. J. Nutr., 111, 602-609.

Singh, R., Kaushik, S., Wang, Y., Xiang, Y., Novak, I., Komatsu, M., Tanaka, K., Cuervo, A.M. and Czaja, M.J. (2009): Autophagy regulates lipid metabolism. Nature, 458, 1131-1135.

Takahashi, Y., Shinoda, A., Furuya, N., Harada, E., Arimura, N., Ichi, I., Fujiwara, Y., Inoue, J. and Sato, R. (2013): Perilipin-mediated lipid droplet formation in adipocytes promotes sterol regulatory element-binding protein-1 processing and triacylglyceride accumulation. PLoS One, 8, e64605.

Theise, N.D. (2014): Liver and Gallbladder. In: Robbins and Cotran Pathologic basis of disease 9th ed. (Kumar, V., Abbas, A.K. and Aster, J.C., eds.), pp.821-881, Elsevier Health Sciences, Philadelphia.

Thier, R., Wiebel, F.A., Hinkel, A., Burger, A., Brüning, T., Morgenroth, K., Senge, T., Wilhelm, M. and Schulz, T.G. (1998): Species differences in the glutathione transferase GSTT1-1 activity towards the model substrates methyl chloride and dichloromethane in liver and kidney. Arch. Toxicol., 72, 622-629.

Umezu, T., Nakamiya, K., Kita, K., Ochi, T., Shibata, Y. and Morita, M. (2012): Diphenylarsinic acid produces behavioral effects in mice relevant to symptoms observed in citizens who ingested polluted well water. Neurotoxicol. Teratol., 34, 143151 .

Valko, M., Morris, H. and Cronin, M.T. (2005): Metals, toxicity and oxidative stress. Curr. Med. Chem., 12, 1161-1208.

Vdoviaková, K., Petrovová, E., Krešáková, L., Maloveská, M., Teleky, J., Jenčová, J., Živčák, J. and Jenča, A. Jr. (2016): Importance rat liver morphology and vasculature in surgical research. Med. Sci. Monit., 22, 4716-4728.

Xie, Y., Trouba, K.J., Liu, J., Waalkes, M.P. and Germolec, D.R. (2004): Biokinetics and subchronic toxic effects of oral arsenite, arsenate, monomethylarsonic acid, and dimethylarsinic acid in v-Ha-ras transgenic (Tg.AC) mice. Environ. Health Perspect., 112, 1255-1263.

Yamaguchi, T., Gi, M., Yamano, S., Fujioka, M., Tatsumi, K., Kawachi, S., Ishii, N., Doi, K., Kakehashi, A. and Wanibuchi, H. (2017): A chronic toxicity study of diphenylarsinic acid in F344 rats in drinking water for 52 weeks. Exp. Toxicol. Pathol., 69, 1-7.

Yu, Y., Ping, J., Chen, H., Jiao, L., Zheng, S., Han, Z.G., Hao, P. and Huang, J. (2010): A comparative analysis of liver transcriptome suggests divergent liver function among human, mouse and rat. Genomics, 96, 281-289. 